\title{
Nutritional Menu Planning: A Hybrid Approach and Preliminary Tests
}

\author{
Oscar Chávez-Bosquez ${ }^{1}$, Jerusa Marchi $^{2}$, and Pilar Pozos-Parra ${ }^{1}$ \\ 1 Universidad Juárez Autónoma de Tabasco, División Académica de Informática y \\ Sistemas. Tabasco, \\ Mexico \\ 2 Universidade Federal de Santa Catarina, SC, \\ Brazil \\ \{oscar.chavez, pilar.pozos\}@ujat.mx, jerusa.marchi@ufsc.br
}

\begin{abstract}
Menu planning is a process appearing to be straightforward but many complexities arise when it is tried to be solved by computer means. Actually, although there is evidence of previous work since 50 years ago, at present there is no wide know tool which can solve this task in an automated manner. Also, not all proposals deal with full recipes along with considering the user food preferences. In this paper we propose a system architecture based on hybrid optimization: a first module based on mathematical programming, a well known robust approach to this problem; and a second module based on belief merging, a lesser known framework aimed to combine the nutrition scientist advices and policies along with the user food desires. The association of numerical and symbolic approaches will allow us to generate of a more agreeable menu. In order to illustrate our proposal, we present a motivating example detailing the main aspects of the system.
\end{abstract}

Keywords: Diet planning, menu planning, knowledge representation, belief merging, laws of nutrition.

\section{Introduction}

A good nutrition is the one that provides to the body the correct amount of energy and the necessary nutrients to maintain the vital functions of an individual and to perform his/her daily activities. Human beings ingest energy and nutrients in the form of a diet consisting of different foods. Dietitians and nutritionist are the scientists who plan food and meal preparations, promote healthy eating habits and help patients with specific nutrition needs to get the essential nutrients for them.

As there is no instruction guide with the steps to generate menus, nor a generic accepted criteria to classify a produced menu as good or bad, we propose to build a model based on the called "Laws of Nutrition" or "Laws of Feeding": 
Law of Quantity, Law of Quality, Law of Harmony, Law of Adequacy [7. This set of rules were proposed by Pedro Escudero, which is considered the "father of nutrition" in Latin America [142317.

We enforce two of the four laws in our proposal:

Law of Quantity. Individual's food intake must supply the amount of calories his/her body needs. This value is commonly obtained via anthropometric measurements of the user, mainly gender, age, weight and height; in addition to physical activity. For flexibility, a diet can supply $\pm 10 \%$ of the user's caloric requirement.

Law of Adequacy. The diet must be adequate to each user according not only to his nutritional requirements, but also to his both social and physiological needs. This means that the diet must consider intolerance and preference of aliments. This Law is focused on the final user. For example, it can set restrictions such as cultural manners, climate topics, or geographical issues.

Due to the above restrictions, planning an adequate nutritious menu for individuals and groups is a complex task. There have been multiple attempts to solve it using mathematical algorithms via computational ways since the decade of $60 \mathrm{~s}$ [18]. Since then, there have intensive research in the field, from solutions ranging from mathematical programming $([15 / 26])$ to expert systems ([229]) to evolutionary computing and metaheuristics [10[13].

Most of these solutions work mainly with numerical values of the food: amount of calories, carbohydrates, protein and lipids; some proposals consider the food cost. However, the user choices, preferences, acquired tastes or dietary habits usually are not taken into account, since the computational solutions are implemented from the point of view of the nutrition scientist, for whom the goal is mainly to prescribe healthy foods.

Therefore, we propose a hybrid approach to take into account the user desires and preferences in order to generate a menu more according to the user's point of view, and consequently more acceptable by the user. This inclusion will be done via Belief Merging, a framework used for the integration of knowledge from different sources modeled as propositional formulae.

Finally, some of the approaches work only with basic food items, letting to the nutrition scientist the responsibility of choose the items forming the recipes. In our proposal, we work with basic food items as well with full recipes in order to give a personalized diet considering the user preferences.

\section{Belief Merging}

Belief merging aims at combining several pieces of (possibly inconsistent) information coming from different sources [12. The goal is to produce a single consistent set of information, trying to keep the most of the information of the sources.

Belief merging is an important issue in artificial intelligence and databases, and its applications are many and diverse [4. For example, in multiagent systems 
a merging operator defines the beliefs of a group of agents according to the beliefs of each member of the group. When agents have conflicting beliefs about the "true" state of the world, belief merging can be used to determine the "true" state of the world for the group [24].

A belief merging operator is the responsible for making the belief merging. Several merging operators have been defined and characterized in a logical way. Among them, PS-Merge is a versatile operator which can be used to real-world problems, although only demonstrated solving "toy examples" due to its exponential complexity [6].

Preliminary concepts. In this theory we consider a language $\mathcal{L}(P)$ of propositional logic using a finite ordered set of symbols $P=\left\{p_{1}, p_{2}, \ldots, p_{n}\right\}$ where the formulae are in Disjunctive Normal Form (DNF). A formula $v$ is in DNF iff $v$ is a disjunction of terms $v=D_{1} \vee \ldots \vee D_{m}$, where each term $D_{i}$ is a conjunction of literals $D_{i}=l_{1} \wedge \ldots \wedge l_{k}$, with $l_{i}=p_{j}$ or $l_{i}=\neg p_{j}$.

A belief base or knowledge base (KB) $K$ is a finite set of propositional formulae of $\mathcal{L}$ representing the beliefs from a source. We identify $K$ with the conjunction of its elements.

An interpretation, or state, or world is a function $w$ from $P$ to $\{1,0\}$. These values are identified with the classical truth values true and false respectively. The set of all possible interpretations will be denoted as $\mathcal{W}$, its elements are denoted by vectors of the form $\left(w\left(p_{1}\right), \ldots, w\left(p_{n}\right)\right)$. A model of a propositional formula $v$ is an interpretation such that $w(Q)=1$ once $\mathrm{w}$ is extended in the usual way over the connectives, and the set of models of a formula $v$ will be denoted by $\bmod (v) . K$ is consistent if there exists a model of $K$.

If $v$ is a propositional formula or a set of propositional formulae then $\mathcal{P}(v)$ denotes the set of atoms appearing in $v .|P|$ denotes the cardinality of set $P$. A literal is an atom or its negation.

A belief profile $E=\left\{K_{1}, \ldots, K_{m}\right\}$ is a multiset (bag with possible repeated elements) of $m$ belief bases.

$\boldsymbol{P S}$-Merge Operator. This belief merging operator is an alternative way to distance-based operators [11161825] or to syntax-based operators [219], as it is based on the notion of Partial Satisfiability [5]. PS-Merge produces similar results to other merging approaches, but while other approaches require many merging operators in order to achieve satisfactory results for different scenarios, this approach obtains similar results for all these different scenarios with a unique operator 24].

Let $E=\left\{K_{1}, \ldots, K_{m}\right\}$ be a belief profile and $P S$-Merge be a function which maps a belief profile to a belief base, PS-Merge : $\mathcal{L}(P)^{n} \rightarrow \mathcal{L}(P)$, then the Partial Satisfiability Merge of $E$ is $P S$-Merge $(E)$ such that the set of models of the resulting base is:

$$
\left\{w \in \mathcal{W} \mid \sum_{i=1}^{m} w_{p s}\left(K_{i}\right) \geq \sum_{i=1}^{m} w_{p s}^{\prime}\left(K_{i}\right) \text { for all } w^{\prime} \in \mathcal{W}\right\}
$$


Let $K$ be a belief base, $w$ any interpretation of $\mathcal{W}$ and $|P|=n$, we define the Partial Satisfiability of $K$ for $w$, denoted as $w_{p s}\left(Q_{K}\right)$, as follows:

- if $Q_{K}:=C_{1} \wedge \cdots \wedge C_{s}$ where $C_{i}$ are literals then:

$$
w_{p s}\left(Q_{K}\right)=\max \left\{\sum_{i=1}^{s} \frac{w\left(C_{i}\right)}{s}, \frac{n-\left|\mathcal{P}\left(\bigwedge_{i=1}^{s} C_{i}\right)\right|}{2 n}\right\}
$$

- if $Q_{K}:=D_{1} \vee \cdots \vee D_{r}$ where each $D_{i}$ is a literal or a conjunction of literals then:

$$
w_{p s}\left(Q_{K}\right)=\max \left\{w_{p s}\left(D_{1}\right), \ldots, w_{p s}\left(D_{r}\right)\right\}
$$

The intuitive explanation of Partial Satisfiability is as follows [5]: it is natural to think that if we have the conjunction of two literals and just one is satisfied then we are satisfying $50 \%$ of the conjunction. If we generalize this idea we can measure the satisfaction of a conjunction of one or more literals as the sum of their evaluation under the interpretation divided by the number of conjuncts. However, the agent may consider only some atoms of the language, then the agent is not affected by the decision taken over the atoms that are not considered. So, in this case we give a partial satisfaction of $50 \%$ for each atom not appearing in the agent's beliefs. On the other hand, the agent is interested in satisfying the literals that appear in its beliefs, and we interpret this fact by assigning a satisfaction of $100 \%$ to each literal verified by the interpretation and $0 \%$ to those that are falsified.

\section{Proposal}

We present a hybrid approach to solve the menu planning problem, by means of the system architecture shown in Figure 1. Although significant data preprocessing is done, we divide the overall system functionality in two algorithms, each one performing one main task of the process. The system uses different inputs according to each step, whereas outputs are menus at different level of adequation: some menu could have a high level of adequacy for a nutritionist (it meets the user's caloric requirement), but a poor level of adequacy for the final user (it has no favourite foods included).

\subsection{Phase 1}

First, system takes the user caloric needs and the database with all the available foods to generate a first approximation to a menu in accordance with the energy needed by the user. It is worth noticing that this approximation to a menu may correspond to a possibly incorrect diet according to the Law of Adequacy, that's why we name this result as list of foods. Each list of foods contains no element of the user's food intolerance set. 
The proposed menu is divided into meals in a day. User specifies the amount of meals he wants to take in a day, and he can also specify which meals are most important by allocating a percentage of intake per meal. System generates 3 lists of foods per meal according to the user caloric needs: the one closer to the needs $-10 \%$, another one closer to the exact value of the user needs, and the closer to the needs $+10 \%$.

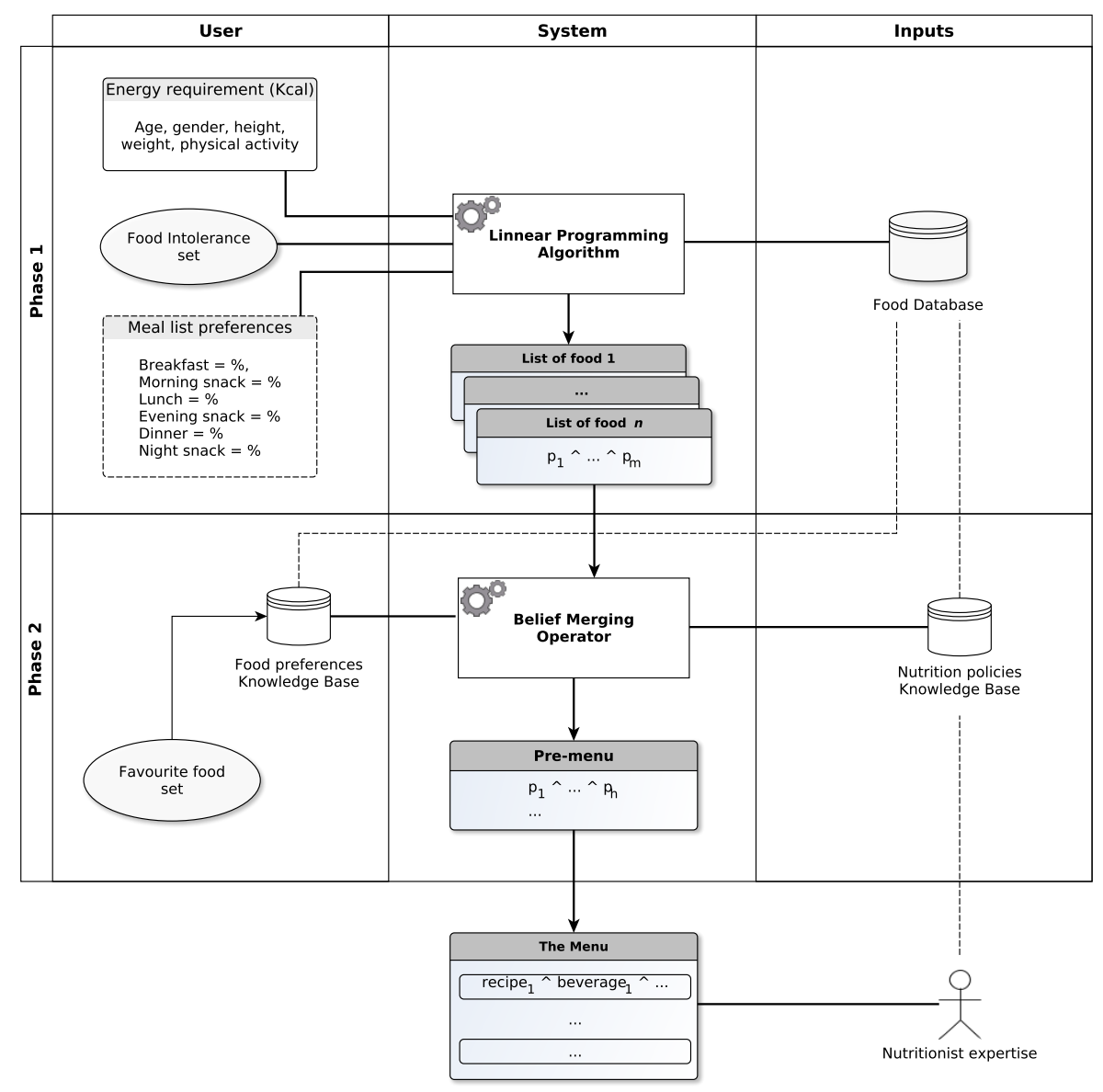

Fig. 1. System architecture. The system (center column) is composed of two algorithms, one for each phase. Input consists of data from the user (first column) and predefined data structures (third column). The final result is an adequate menu.

The main algorithm in this step generates each list of foods via Linear Programming, inspired in the model presented in [21. Thus we had to translate the problem into a mathematical model. The result of this stage is in knowledge 
base format, so we have to translate each food to its corresponding variable.

\subsection{Phase 2}

At this step, the system tries to meet the user preferences by means of a belief merging operator, since all inputs are symbolic data coming from different sources equally respectable but most likely inconsistent.

We start by translating the user food preferences into a knowledge base format. This KB is merged with the lists of food generated in the previous phase and with a KB of nutritional polices, i.e. "common sense" information (for example the adequate combination of foods). The last $\mathrm{KB}$ will be filled up by the nutrition scientist knowledge, but it can contain other kind of information. The main algorithm performing this step is the PS-Merge belief merging operator 24]. The output is expected to be a consistent KB per list of food.

In order to show a human-readable menu, the resulting $\mathrm{KB}$ is translated from propositional variables to foods names, and the final configuration is left to the specialist. This resulting menu meets the user's caloric requirement, as well as it complies with the two Laws of Nutrition considered.

\subsection{Food Database}

Figure 2 shows the relational model of the food database proposed. Main tables are food and patient, whose main relations are about the user's food intolerance set (patient_intolerant_food), user favourite food (patient_favourite_food), and the final menu for the day (menu).

\section{A Motivating Example}

Now we will show a simple instance that illustrates the hybrid approach of our proposal. We will describe the entire process by generating a breakfast, one single meal from a complete menu. Each element of the system architecture will be described in terms of the example instance, emphasizing in the belief merging process, the main contribution of this work.

\subsection{User Data}

Energy Requirement. We need to plan a menu for a 33 year old male, weight $84 \mathrm{~kg}, 186 \mathrm{~cm}$ tall, and an intense physical activity. To obtain his caloric requirement we have to calculate the Basal Metabolic Rate (BMR), which is the minimum amount of energy that the body needs without realizing any activity using the following formula $\underline{3}$ :

$\mathrm{BMR}_{\text {men }}=(10 \times$ weight $)+(6.25 \times$ height $)-(5 \times$ age $)+5=1680 \mathrm{kcal}$

Afterwards, we calculate the Total Energy Expenditure (TEE) representing the amount of energy needed to maintain the vital functions of the body and 
Nutritional Menu Planning: A Hybrid Approach and Preliminary Tests

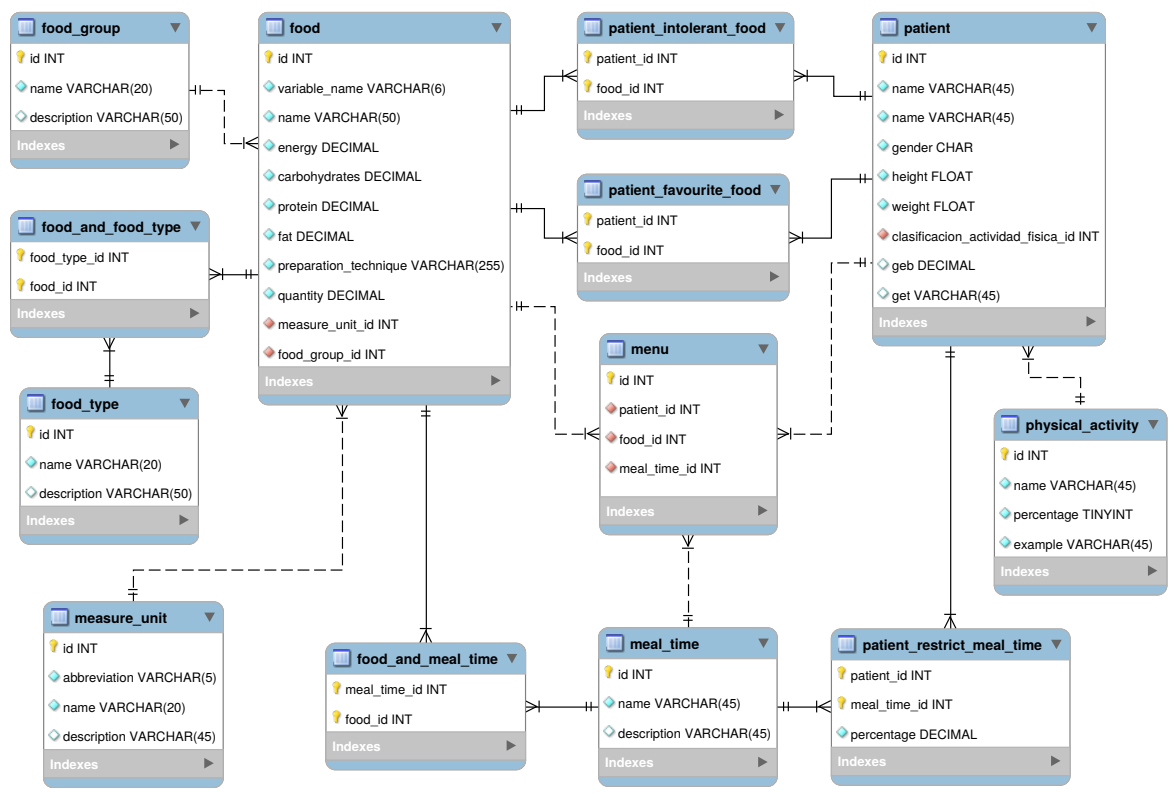

Fig. 2. Foods database relational diagram.

perform the daily activities. This is obtained from the BMR plus two values: adding a $10 \%$ due to the digestive effect (Thermic Effect of the Food, TEF), and adding a percentage depending the user's physical activity (Physical Activity Factor, PAF) according to Repose $=20 \%$, Sedentary $=37.5 \%$, Moderate $=55 \%$, Intense $=72.5 \%$ [3]. So the TEE for this patient is as follows:

$\mathrm{TEE}=\mathrm{BMR}+\mathrm{TEF}+\mathrm{PAF}=1680+168+1218=3066 \mathrm{kcal}$

Food Intolerance. The user claims to be lactose intolerant.

Menu Distribution. The individual can only take three meals per day, so we must distribute all his food intake into three meals, according to the following distribution:

\begin{tabular}{lcr} 
Meal & Percentage & Energy \\
\hline Breakfast & $30 \%$ & $920 \mathrm{kcal}$ \\
Lunch & $40 \%$ & $1226 \mathrm{kcal}$ \\
Dinner & $30 \%$ & $920 \mathrm{kcal}$ \\
\hline
\end{tabular}

Favourite Foods. Apples and bread in the breakfast, and he always eats pepper sauce in each meal. He also dislikes papaya fruit. According to foods shown in Table 1. these beliefs can be modeled in the Food Preferences KB as: 


$$
K_{1}=(a \wedge b \wedge m \wedge \neg p)
$$

\subsection{Input Data Structures}

Food Database. Table 1 considers the foods from the database related to breakfast. Data were obtained from a local nutrition lab. For the sake of space, we only shown the relevant information related to this example.

Table 1. Subset of breakfast foods in database.

\begin{tabular}{clrlllllr}
\hline Var. & Name & Energy & Group & Type & Time & Meal & Unit & Qty. \\
\hline$a$ & Apple & 70 & $\mathrm{~F}$ & $\mathrm{~F}$ & $1,2,3$ & $\mathrm{~B}, \mathrm{~S}, \mathrm{D}$ & Piece & 1 \\
$n$ & Banana & 96 & $\mathrm{~F}$ & $\mathrm{~F}$ & $1,2,3$ & $\mathrm{~B}, \mathrm{~S}, \mathrm{D}$ & Piece & 1 \\
$p$ & Papaya & 43 & $\mathrm{~F}$ & $\mathrm{~F}$ & 1,2 & $\mathrm{~B}, \mathrm{~S}, \mathrm{D}$ & gr & 200 \\
$s$ & Eggs with ham & 310 & $\mathrm{P}$ & $\mathrm{E}$ & 4 & $\mathrm{~B}$ & Portion & 1 \\
$u$ & Lima beans soup & 328 & $\mathrm{P}$ & $\mathrm{C}$ & 4 & $\mathrm{~B}, \mathrm{~L}$ & Portion & 1 \\
$e$ & Eggs with beans & 446 & $\mathrm{P}$ & $\mathrm{E}, \mathrm{L}$ & 4 & $\mathrm{~B}, \mathrm{~L}$ & Portion & 1 \\
$h$ & Hot cakes & 362.5 & $\mathrm{C}$ & $\mathrm{C}$ & 4 & $\mathrm{~B}$ & Portion & 1 \\
$i$ & Cappuccino coffee & 157 & $\mathrm{C}$ & $\mathrm{C}$ & 5 & $\mathrm{~B}, \mathrm{D}$ & Cup & 1 \\
$c$ & Caffè Americano & 21 & $\mathrm{C}$ & $\mathrm{C}$ & 5 & B,D & Cup & 1 \\
$o$ & Orange juice & 197.5 & $\mathrm{~F}$ & $\mathrm{~F}$ & 5 & B & Glass & 1 \\
$k$ & Hot milk & 116 & $\mathrm{P}$ & $\mathrm{K}$ & 5 & B,S & Glass & 1 \\
$b$ & Bread roll & 301 & $\mathrm{C}$ & $\mathrm{C}$ & 2 & B,L,D & Piece & 1 \\
$t$ & Tortilla & 246 & $\mathrm{C}$ & $\mathrm{C}$ & 2 & B,L,D & Piece & 1 \\
$g$ & Green salad & 79.5 & $\mathrm{~V}$ & $\mathrm{~V}$ & 4 & B,S,D & Portion & 1 \\
$l$ & Accompanying salad & 26 & $\mathrm{~V}$ & $\mathrm{~V}$ & 2 & B,L,D & Portion & 1 \\
$m$ & Tomato spicy sauce & 45 & $\mathrm{~V}$ & $\mathrm{~V}$ & 2 & B,L,D & ml & 50 \\
\hline
\end{tabular}

Table 1 uses the following acronyms:

- Type: $\mathrm{C}=$ Cereal, $\mathrm{D}=$ Dairy, $\mathrm{E}=$ Egg, $\mathrm{F}=$ Fruit, $\mathrm{L}=$ Legumes, $\mathrm{M}=$ Meat, $\mathrm{V}=$ Vegetables.

- Group: $\mathrm{C}=$ Cereal, $\mathrm{F}=$ Fruit, $\mathrm{P}=$ Protein, $\mathrm{V}=$ Vegetables.

- Time: 1 = Entrance, 2 = Accompanying, 3 = Dessert, $4=$ Main dish, $5=$ Drink.

- Meal: B = Breakfast, $\mathrm{S}=$ Snack, $\mathrm{L}=$ Lunch, $\mathrm{D}=$ Dinner.

Nutrition Policies KB. For this case, we consider the following expert advises: (i) not to eat bread and tortilla in the same meal, because both are high carbohydrates foods; and (ii) it is recommended to eat apple, orange or papaya in breakfast due to their digestive properties. With the foods shown in Table 1 . these two beliefs can be modeled in the Nutrition Policies KB as:

$$
K_{2}=(\neg(b \wedge t) \wedge(a \vee o \vee p))
$$




\subsection{Phase 1}

In this step we employ Linear Programming to generate the lists of foods. First we get rid of the intolerance foods using a subset from Table 1 without food from dairy type (no hot milk, cappuccino coffee nor hot cakes in this case). Within this subset we find three lists of foods whose energy complies the breakfast's energy required by the user $(920 \mathrm{kcal})$ :

\begin{tabular}{|c|c|}
\hline List of foods & 1 \\
\hline Apple & 70 \\
\hline Eggs with beans & 446 \\
\hline Caffè Americano & 21 \\
\hline Bread roll & 301 \\
\hline Total energy: & 838 \\
\hline
\end{tabular}

\begin{tabular}{lr}
\multicolumn{2}{c}{ List of foods } \\
\hline Papaya & 43 \\
Apple & 70 \\
Eggs with ham & 310 \\
Orange juice & 197.5 \\
Bread roll & 301 \\
\hline Total energy: & 921.5 \\
\hline
\end{tabular}

\begin{tabular}{lr}
\multicolumn{2}{c}{ List of foods $\mathbf{3}$} \\
\hline Green salad & 79.5 \\
Apple & 70 \\
Lima beans soup & 348 \\
Orange juice & 197.5 \\
Bread roll & 301 \\
\hline Total energy: & 996 \\
\hline
\end{tabular}

Energy of the first list of foods is under the breakfast's exact energy requirement, but over the $-10 \%$. Energy of the second list of foods is close to the exact breakfast's energy requirement. Energy of the third list of foods is above the breakfast's energy requiriement but lower than the $+10 \%$.

\subsection{Phase 2}

In this step we employ Belief Merging to generate menus. Translating list of foods 1 as KB:

$$
K_{3}=(a \wedge e \wedge i \wedge b)
$$

Gives us the belief profile to be merged:

$$
E=\left(K_{1}, K_{2}, K_{3}\right)
$$

$P S$-Merge operator requires normalizing each KB to DNF:

$$
\begin{aligned}
& Q_{K_{1}}=(a \wedge b \wedge m \wedge \neg p) \\
& Q_{K_{2}}=(\neg b \wedge a) \vee(\neg b \wedge o) \vee(\neg b \wedge p) \vee(\neg t \wedge a) \vee(\neg t \wedge o) \vee(\neg t \wedge p) \\
& Q_{K_{3}}=(a \wedge e \wedge i \wedge b)
\end{aligned}
$$

$P S$-Merge operator is used to find $\mathrm{KB}$ with no inconsistencies and with most of the food preferences and nutrition policies satisfied. For example, taking $Q_{K_{2}}$ and the interpretation $w^{\prime}=(1,1,1,1,1,1,0,0)$ corresponding to the propositional values $(w(a), w(b), w(e), w(i), w(m), w(o), w(p), w(t))$ in that order, we have:

$$
\begin{gathered}
w_{p s}^{\prime}\left(Q_{K_{2}}\right)=\max \left\{w_{p s}^{\prime}(\neg b \wedge a), w_{p s}^{\prime}(\neg b \wedge o), w_{p s}^{\prime}(\neg b \wedge p), w_{p s}^{\prime}(\neg t \wedge a),\right. \\
\left.w_{p s}^{\prime}(\neg t \wedge o), w_{p s}^{\prime}(\neg t \wedge p)\right\}
\end{gathered}
$$


Obtaining the partial satisfiability of the first element:

$$
\begin{aligned}
w_{p s}^{\prime}(\neg b \wedge a) & =\max \left\{\frac{w^{\prime}(\neg b)+w(a)}{2}, \frac{8-|\mathcal{P}(\neg b, a)|}{2(8)}\right\}=\max \left\{\frac{0+1}{2}, \frac{8-2}{16}\right\} \\
& =\max \left\{\frac{1}{2}, \frac{3}{8}\right\}=\frac{1}{2}
\end{aligned}
$$

Computing the partial satisfiability of the remaining elements we can obtain the partial satisfiability of the KB with respect to $w^{\prime}$ :

$$
w_{p s}^{\prime}\left(Q_{K_{2}}\right)=\max \left\{\frac{1}{2}, \frac{1}{2}, \frac{3}{8}, 1,1, \frac{1}{2}\right\}=1
$$

For the sake of space and clarity, below we only show the interpretations corresponding to the best partial satisfiability. Both $w_{1}$ and $w_{2}$ contain the views of the majority, representing the best possible menu according to the input KBs:

Belief merging result via PS-Merge

\begin{tabular}{ccccccccccccc}
\hline & $a$ & $b$ & $e$ & $i$ & $m$ & $o$ & $p$ & $t$ & $w_{p s}\left(Q_{K_{1}}\right)$ & $w_{p s}\left(Q_{K_{2}}\right)$ & $w_{p s}\left(Q_{K_{3}}\right)$ & Sum \\
$w_{1}$ & 1 & 1 & 1 & 1 & 1 & 1 & 0 & 0 & 1 & 1 & 1 & 3.0 \\
$w_{2}$ & 1 & 1 & 1 & 1 & 1 & 0 & 0 & 0 & 1 & 1 & 1 & 3.0 \\
\hline
\end{tabular}

The final process of this stage is to translate these KBs to menus, and then verify if the menu is still consistent according to the energy requirement of the user:

\begin{tabular}{lr}
\multicolumn{2}{c}{ Menu 1 } \\
\hline Apple & 70 \\
Eggs with beans & 446 \\
Caffè Americano & 21 \\
Bread roll & 301 \\
Tomato spicy sauce & 45 \\
Orange juice & 197.5 \\
\hline Total energy: & 1080.5 \\
\hline
\end{tabular}

\begin{tabular}{lr}
\multicolumn{2}{c}{ Menu 2 } \\
\hline Apple & 70 \\
Eggs with beans & 446 \\
Caffè Americano & 21 \\
Bread roll & 301 \\
Tomato spicy sauce & 45 \\
\hline Total energy: & 883 \\
\hline
\end{tabular}

We can notice that energy in Menu 1 is above the user's needs, so this menu must be discarded. On the other hand, Menu 2 has a right amount of energy, as well as satisfies the user's food preferences and also complies with the nutrition policies.

Lastly, the adequate menu, or maybe a set of menus, are presented to the nutrition scientist, who will choose or modify the proposals to finally prescribe the diet to the user.

\section{Conclusions}

Diet planning is considered as an art, but we consider the need of a set of strict rules to give a reference of the "correctness" of a menu. In order to produce 
adequate menus, we use the "Laws of Nutrition" as a formal reference to validate our results. So, our proposal enforces the Law of Quantity as well as the Law of Adequacy, the latter with emphasizing the user preferences.

We have presented a novel approach for dealing with the menu planning problem. The proposed architecture is based on two formal frameworks used in two phases to solve the different views of the diet planning problem.

In the first step, the Linear Programming method has been proven to solve the problem properly and on time. For this purpose, we focus on build a mathematical model containing the quantitative information of the problem. In this way we obtain menus in accordance with the user's nutritional needs.

In the second step, we implement a Belief Merging operator, a relatively new framework which few applications to real-world problems are known (i.e. [20]). For this purpose, we focus on build a logical model containing the qualitative information of the problem. In this way we obtain menus in accordance with the user's food desires. PS-Merge belief merging operator provides good results, as it tries to satisfy the majority opinion and so minimize the level of total dissatisfaction.

We have shown that this hybrid proposal can be a viable approach to solve the problem at a very specific level. Further research is required to provide evidence of the effectiveness and feasibility of this proposal to complete menus with bigger input data.

\section{References}

1. Balintfy, J.L.: Menu planning by computer. Communications of the ACM 51(6), 255-259 (1964)

2. Baral, C., Kraus, S., Minker, J., Subrahmanian, V.: Combining knowledge bases consisting of first order theories. In: Ras, Z., Zemankova, M. (eds.) Methodologies for Intelligent Systems, Lecture Notes in Computer Science, vol. 542, pp. 92-101. Springer Berlin Heidelberg (1991)

3. Berman, R.: Boosting Your Metabolism For Dummies. EBL ebooks online, Wiley (2013)

4. Bloch, I., Hunter, A.: Fusion: General concepts and characteristics. International Journal of Intelligent Systems 10(16), 1107-1134 (2001)

5. Borja Macías, V., Pozos Parra, P.: Model-based belief merging without distance measures. In: Proceedings of the 6th International Joint Conference on $\mathrm{Au}-$ tonomous Agents and Multiagent Systems. pp. 154:1-154:3. AAMAS '07, ACM, New York, NY, USA (2007)

6. Borja Macías, V., Pozos Parra, P.: Implementing PS-Merge Operator. In: Hernández Aguirre, A., Monroy Borja, R., Reyes García, C. (eds.) MICAI 2009: Advances in Artificial Intelligence, Lecture Notes in Computer Science, vol. 5845, pp. 39-50. Springer Berlin Heidelberg (2009)

7. Deon, B.C., Medeiros, L.B., de Freitas Saccol, A.L., Hecktheuer, L.H., Saccol, S., Naissinger, M.: Good food preparation practices in households: A review. Trends in Food Science \& Technology (In press) (2014)

8. Eckstein, E.F.: Menu planning by computer: The random approach. Journal of the American Dietetic Association 51(6), 529-533 (1967) 
9. Ejtahed, H.S., Sarsharzadeh, M.M., Mirmiran, P., Asghari, G., Yuzbashian, E., Azizi, F.: Leemoo, a dietary assessment and nutritional planning software, using fuzzy logic. International Journal of Endocrinology Metabolism 11(4), e10169 (2013)

10. Gaál, B., Vassányi, I., Kozmann, G.: A novel artificial intelligence method for weekly dietary menu planning. Methods of Information in Medicine 44(5), 655-664 (2005)

11. Konieczny, S., Pino Pérez, R.: On the logic of merging. In: Sixth International Conference on Principles of Knowledge Representation and Reasoning (KR'98). pp. 488-498 (1998)

12. Konieczny, S., Pino Pérez, R.: Logic based merging. Journal of Philosophical Logic 40(2), 239-270 (2011)

13. Koroušić Seljak, B.: Computer-based dietary menu planning. Journal of Food Composition and Analysis 22(5), 414-420 (2009), 7th International Food Data Conference: Food Composition and Biodiversity

14. Kott, S., Droux, J.: Globalizing Social Rights: The International Labour Organization and Beyond. International Labour Organization (ILO) Century Series, Palgrave Macmillan (2013)

15. Leung, P., Wanitprapha, K., Quinn, L.: A recipe-based, diet-planning modelling system. British Journal of Nutrition 74, 151-162 (8 1995)

16. Liberatore, P., Schaerf, M.: Arbitration (or how to merge knowledge bases). IEEE Transactions on Knowledge and Data Engineering 10(1), 76-90 (1998)

17. Library, U.H.S.: Pedro Escudero. http://hsl.lib.unc.edu/ specialcollections/bios/escudero (2013)

18. Lin, J., Mendelzon, A.O.: Knowledge Base Merging by Majority. In: Pareschi, R., Fronhoefer, B. (eds.) Dynamic Worlds: From the Frame Problem to Knowledge Management. Kluwer (1999)

19. Marchi, J., Bittencourt, G., Perrussel, L.: Prime forms and minimal change in propositional belief bases. Annals of Mathematics and Artificial Intelligence 59(1), 1-45 (2010)

20. McAreavey, K., Liu, W., Miller, P., Meenan, C.: Tools for finding inconsistencies in real-world logic-based systems. In: STAIRS. pp. 192-203 (2012)

21. Papadimitriu, C.: Computational complexity. Addison-Wesley, Reading,Massachusetts (1994)

22. Petot, G.J., Marling, C., Sterling, L.: An artificial intelligence system for computerassisted menu planning. Journal of the American Dietetic Association 98(9), 10091014 (1998)

23. Pite, R.: Creating a Common Table in 20th-century Argentina. University of North Carolina Press (2013)

24. Pozos Parra, P., Borja Macías, V.: Partial satisfiability-based merging. In: Gelbukh, A., Kuri Morales, Ángel Fernando. (eds.) MICAI 2007: Advances in Artificial Intelligence, Lecture Notes in Computer Science, vol. 4827, pp. 225-235. Springer Berlin Heidelberg (2007)

25. Revesz, P.: On the semantics of arbitration. International Journal of Algebra and Computation 7(02), 133-160 (1997)

26. Sklan, D., Dariel, I.: Diet planning for humans using mixed-integer linear programming. British Journal of Nutrition 70 (1993) 